\title{
Surgical Management of a Juvenile Cystic Adenomyoma: A Unique Presentation of Adenomyosis
}

\author{
Huda Afaneh, $M D^{1^{*}}$, Karen Moses, $M D^{1}$, Omari Young, $M D^{2}$ and Mostafa Abuzeid, \\ MD, FACOG, FRCOG $3,4,5$
}

${ }^{1}$ Clinical Instructor, Department of Obstetrics and Gynecology, Hurley Medical Center, Michigan State University College of Human Medicine, USA

${ }^{2}$ Assistant Professor, Department of Obstetrics and Gynecology, IVF Michigan Rochester Hills and Flint PC, Hurley Medical Center, Michigan State University College of Human Medicine, USA

${ }^{3}$ Director, Reproductive Endocrinology and Infertility Hurley Medical Center, USA

${ }^{4}$ Professor, Michigan State University College of Human Medicine, USA

${ }^{5}$ Practice and Medical Director, IVF Michigan Rochester Hills and Flint PC, USA

*Corresponding author: Huda Afaneh, Clinical Instructor, Department of Obstetrics and Gynecology, Hurley Medical Center, Michigan State University College of Human Medicine, Flint MI, USA

\section{Introduction}

Endometriosis is characterized by the presence of endometrial glands and stroma in ectopic locations within the peritoneal cavity. More commonly, the lesions can be found on areas such as the cul-de-sac and other pelvic parietal surfaces. Endometrial glands and stroma infiltrating the myometrium characterizes Adenomyosis $[1,2]$. Adenomyosis is usually characterized by diffuse foci of endometrial glands scattered throughout the myometrium. Meanwhile, a less common type of adenomyosis, in which the lesions are focal or localized in nature is referred to as anadenomyoma $[3,4]$. An adenomyoma is described as a circumscribed nodule of hypertrophic and distorted endometrium within the myometrium $[3,5]$. Only a few cases in the literature have been reported with histologically confirmed endometrioma within the myometrium of the uterus $[4,6]$. Such patients may primarily present with chronic pelvic pain among the wide spectrum of clinical syndromes in patients with endometriosis. On the other hand, there are a few case reports in the literature describing juvenile cystic adenomyoma as a unique uterine pathology $[4,6,7]$. Juvenile cystic adenomyoma is basically an adenomyoma with a cystic component that occurs in young patients. In this case report, we focus on the surgical management of a juvenile cystic adenomyoma and endometrioma within the uterine wall. The surgical techniques used to excise the cystic adenomyoma closely resemble the techniques used during a myomectomy along with unique surgical challenges when considering the presence of underlying adenomyosis.

\section{Case}

Our patient is a 16 -year-old, gravida 0 , with a history of chronic pelvic pain and dysmenorrhea. Alternative etiology of her pain was ruled out including an infectious, genitourinary and thorough gastrointestinal workup. She did not attempt treatment with hormonal therapy prior to surgery. Initial CT scan of abdomen/pelvis performed prior to surgery suggested a cystic mass separate from the endometrial cavity measuring 2.2 $\mathrm{cm} \times 2.2 \mathrm{~cm}$. Upon initial diagnostic laparoscopy, she was found to have two small endometriotic implants on the left ovary as well as an endometrioma of the uterus diagnosed via needle aspiration. Pathologic evaluation described the aspirated fluid as containing "hemosiderin-laden macrophages and blood, with no evidence of malignancy". She also had a hormonal intrauterine

Citation: Afaneh H, Moses K, Young O, Abuzeid M (2020) Surgical Management of a Juvenile Cystic Adenomyoma: A Unique Presentation of Adenomyosis. Obstet Gynecol Cases Rev 7:162. doi. org/10.23937/2377-9004/1410162

Accepted: April 01, 2020: Published: April 03, 2020

Copyright: (c) 2020 Afaneh $\mathrm{H}$, et al. This is an open-access article distributed under the terms of the Creative Commons Attribution License, which permits unrestricted use, distribution, and reproduction in any medium, provided the original author and source are credited. 


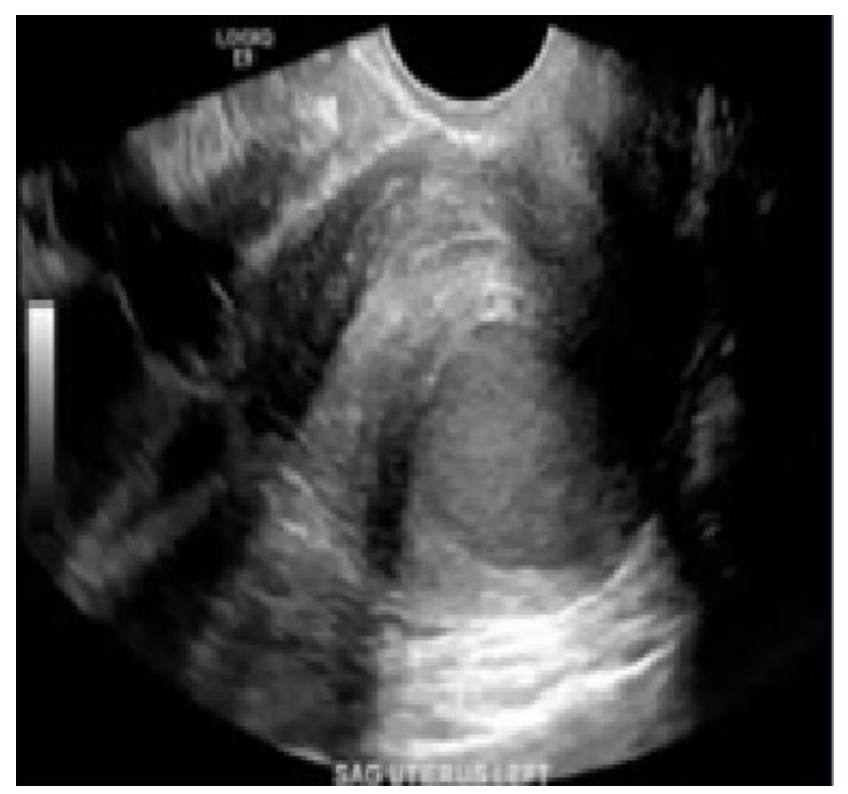

Figure 1: Ultrasound view of juvenile cystic adenomyoma.

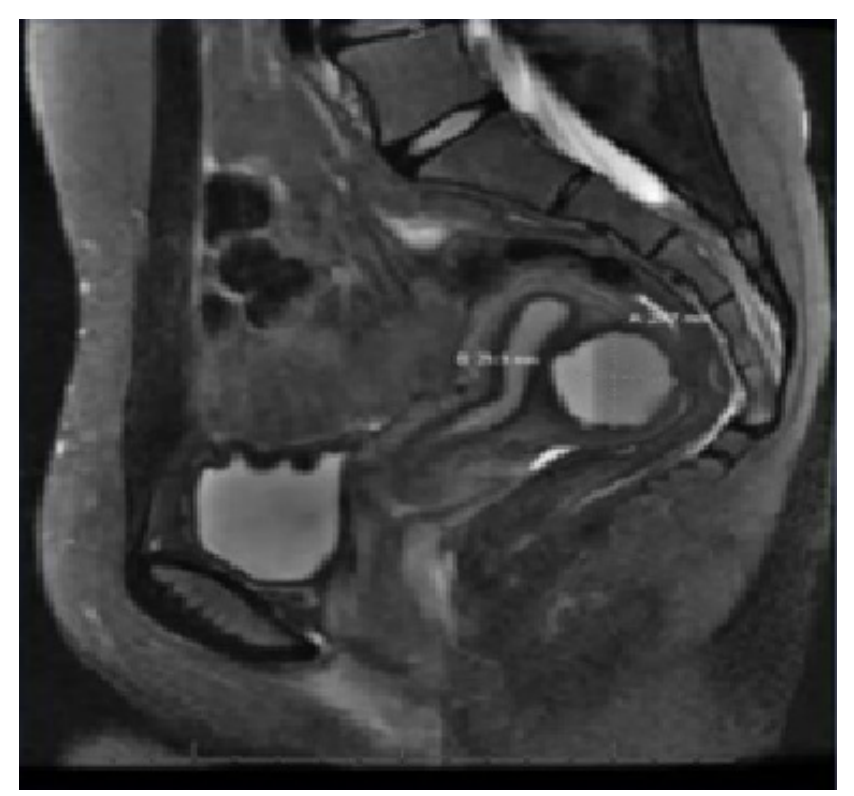

Figure 2: MRI view of juvenile cystic adenomyoma.

device placed intraoperatively. About one month following her surgery, her pain recurred. Repeat imaging with transvaginal ultrasound scan (TVUS) and MRI (Figure 1 and Figure 2) suggested recurrence of her endometrioma. MRI suggested a clearly demarcated approximately $3 \mathrm{~cm} \times 3 \mathrm{~cm}$ mass located within the myometrium. Decision was made for a second surgery in conjunction with a Reproductive Endocrinology and Infertility specialist. On second laparoscopy, the uterine endometrioma was excised and techniques included the use of monopolar energy, sharp and blunt dissection (Figure 3 and Figure 4). Chocolate-like material was noted coming out of the cavity of the endometrioma (Figure 3). The uterine cavity was ultimately not entered (Figure 5). Repair of the defect in the myometrium was closed in a two-layer closure using 2-0 and 3-0 V-lock suture (Figure 6). The

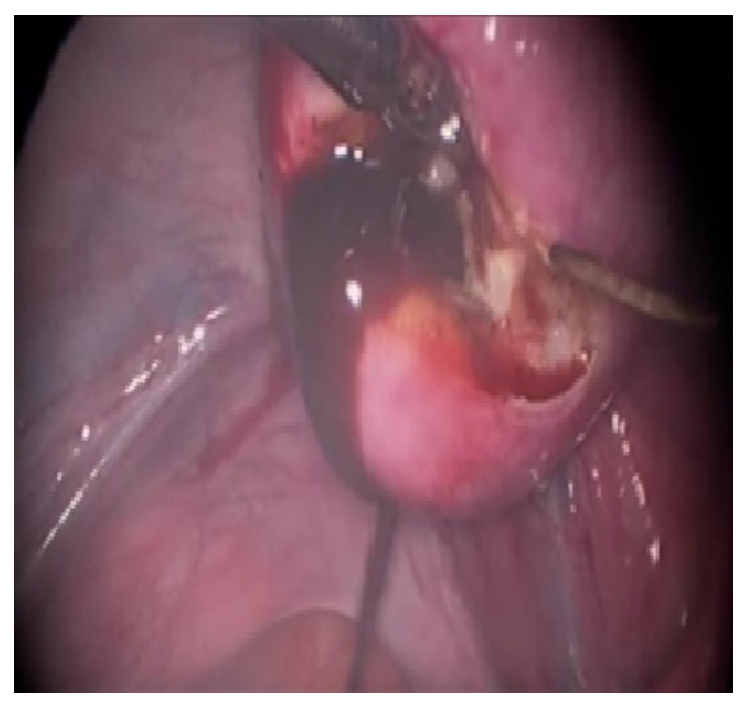

Figure 3: Chocolate-like material exiting from the juvenile cystic adenomyoma encased on the posterior aspect of uterus.

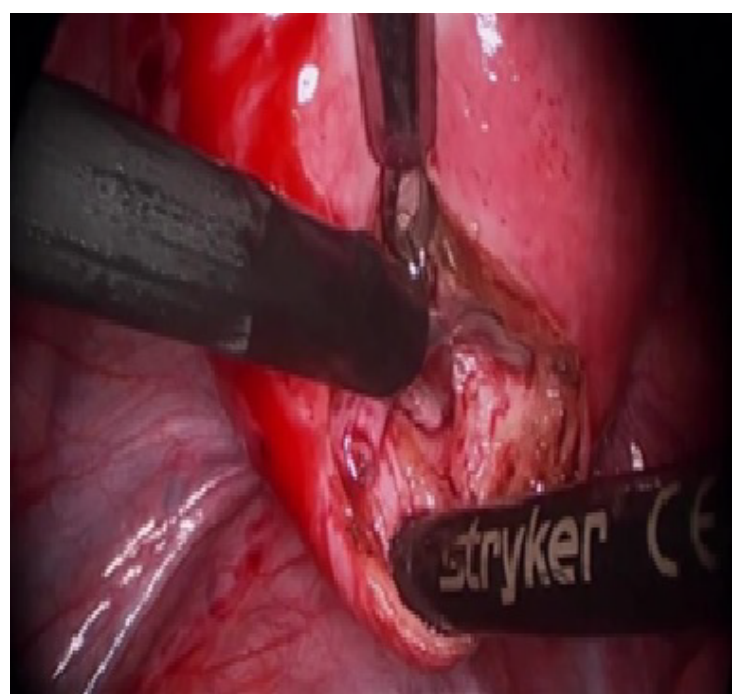

Figure 4: Excision of juvenile cystic adenomyoma.

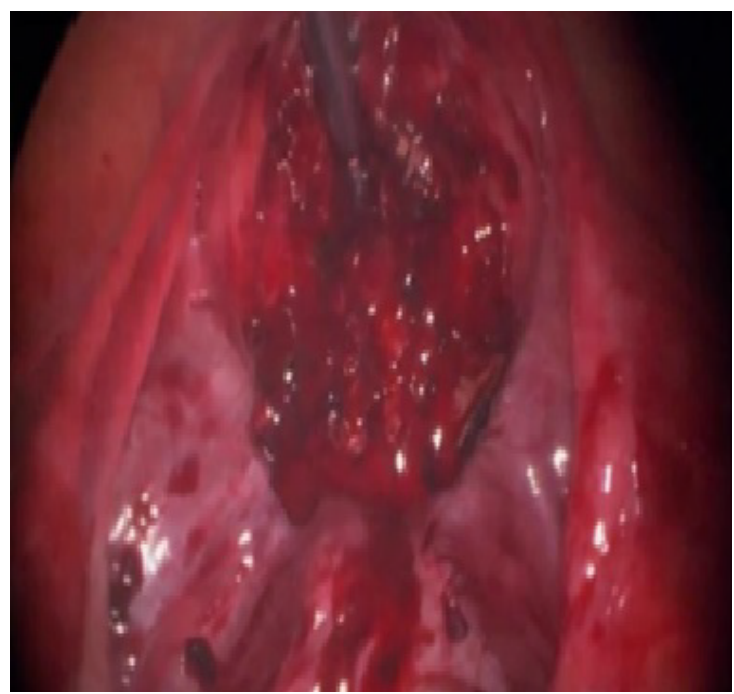

Figure 5: Adenomyoma cyst bed, following excision, with no evidence of entry of the endometrial cavity. 


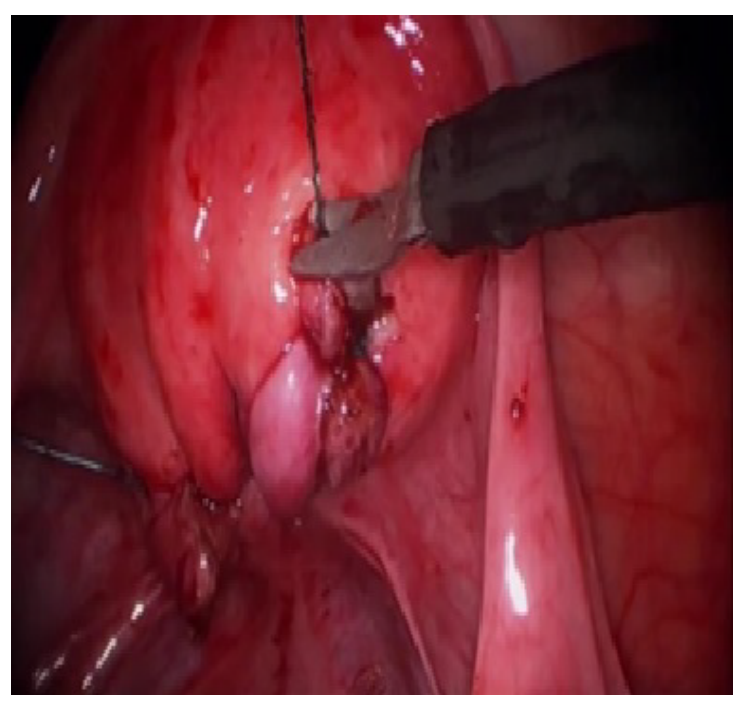

Figure 6: Two-layer repair was carried 2-0 V-lock suture.

duration of surgery was 90 minutes and the estimated blood loss was approximately $40 \mathrm{cc}$. The patient was discharged home on postoperative day 1 and her post-operative period was uneventful. The pathology report confirmed the presence of fragments of myometrium and adenomyosis. Therefore, the diagnosis of juvenile cystic adenomyoma was considered as a primary diagnosis. Post-operatively, the patient did have relief of her pain. Six weeks later, repeat transvaginal ultrasound revealed no evidence of recurrence of the juvenile cystic adenomyoma. She had a follow-up appointment 8 months following her second surgery, and she continues to have relief of her previous symptoms.

\section{Discussion}

Juvenile cystic adenomyoma of the uterus is a rare entity of focal adenomyoma that occurs in young patients. It usually causes severe and debilitating pain. The first report describing juvenile cystic adenomyoma was published by Tamura M, et al. in 1996 [8]. Takeuchi $\mathrm{H}$, et al. (2010) indicated that 30 cases of juvenile cystic adenomyoma (including those in their series) have been reported in the Japanese-language publications [5]. They suggested the following diagnostic criteria: "1) age < 30 years; 2) Cystic lesion of
$<1 \mathrm{~cm}$ in diameter independent of the uterine lumen and covered by hypertrophic myometrium on diagnostic images; and 3) associated with severe dysmenorrheal" [5]. Kriplani A, et al. (2011) described laparoscopic management of 4 patients of juvenile cystic adenomyoma and reviewed the other case reports on the topic [3]. Our case report and supplementary video aims to increase awareness for such an unusual pathology and options for minimally invasive surgical management. In view of a report of 2 patients who had rupture uterus at 32 and 37 week gestation, meticulous uterine closure and avoid use of cautery should be emphasized [9]. In addition, such patients should be managed with maternal Fetal Medicine specialist when pregnant with a plan for an elective cesarean section.

\section{References}

1. Pelvic Mass. In: Hoffman BL, Schorge JO, Bradshaw KD, Halvorson LM, Schaffer JI, et al. Williams Gynecology. ( $3^{\text {rd }}$ edn), McGrawHill, New York.

2. Endometriosis. In: Jones Howard, Rock JA (2015) TeLindes Operative Gynecology. ( $11^{\text {th }}$ edn), Lippincott, Williams \& Wilkins, Philadelphia.

3. Kriplani A, Mahey R, Agarwal N, Bhatla N, Yadav R, et al. (2011) Laparoscopic management of juvenile cystic adenomyoma: Four cases. JMIG 18: 343-348.

4. Osama Zaghmout, Omar Abuzeid, John Hebert, Mostafa Abuzeid (2017) Endometrioma embedded within the myometrium. American Journal of Obstetrics \& Gynecology (AJOB) 34.

5. Takeuchi H, Kitade M, Kikuchi I, Kumakiri J, Kuroda K, et al. (2010) Diagnosis, laparoscopic management, and histopathologic findings of juvenile cystic adenomyoma: A review of nine cases. Fertil Steril 94: 862-868.

6. Abhishek Trehan (2014) Endometrioma contained within the broad ligament. BMJ Case Rep.

7. Younes G, Tulandi T (2018) Conservative surgery for adenomyosis and results: A systematic review. JMIG 25: 265276.

8. Tamura M, Fukaya T, Takaya R, Ip CW, Yajima A (1996) Juvenile adenomyotic cyst of the corpus uteri with dysmenorrhea. Tohoku J Exp Med 178: 339-344.

9. Koo YJIK, Kwon YS (2011) Conservative surgical treatment combined with $\mathrm{GnRH}$ agonist in symptomatic uterine adenomyosis. Pak J Med Sci 27: 365-370. 